\title{
A-B [onaparte]
}

Anisodactylorum 1854 


\section{HARVARD UNIVERSITY.}

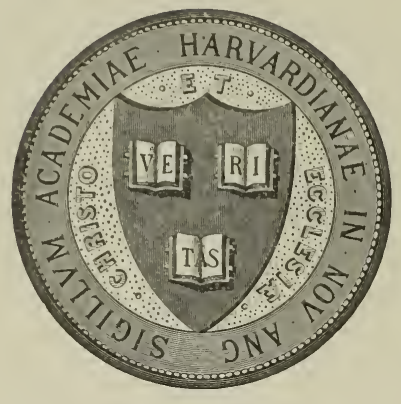

L I B R A R Y

OF THE

MUSEUM OF COMPARATIVE ZOÖLOGY. 30,558

GIFT OF

Mre. Idenry Bryant

December 18,1907. 


\section{0,558}

GONSPECTUS

\section{VOLUCRUII ANISODACTYLORUM.}

AUCTORE

Carolo Luciano Bovapante.

\section{QUADRO DEI VOLUCRI ANISODATTILI.}

L'accoglienza che vi piacque di fare al Quadro de' miei $V_{0-}$ lucri zigodattili mi rende grato di offerirvi quello dei Volucri anisodattili, ciò è a dire di quei Passer che hanno piedi gressori, $o$ ambulatori senza appartenere alla tribù dei Cantori .

Numerosa troppo per una sola Nemoria è questa coorte che vanta più di quattrocento generi, e quasi mille ottocento specie, quindi non comprenderemo in questo scritto l'antipenultima sua stirpe dei Trochili già pubblicata altrove. Le due ultime stirpi dei Cipseli e Notturni saranno da noi riservate per un lavoro comparativo fra esse e le Rondini, ossia veri Fissirostres dei Cantori. Così pure le numerosissime famiglie dei Mioteridi degli Arabatidi e dei Todidi saranno oggetto di speciale pubblicazione, allorquando avrò visitato le Raccolte del celebre ornitologo Lafresnaye in Falaise, le quali ne sono ricchissime.

Comincieremo dunque dalla stirpe dei Frugivori e in questa dai Bucerotidi che tanto ben rappresentano nell'antico mondo gli americani Ranfastidi per lo enorme becco, e per la determinata ubicazione. Conseguitano gli africani Musofagidi e Coliidi rappresentati in America dall'Opistocomido (che come i primi richiama Colombi e Gallinaces) e dai Fitotomidi similissimi ai Tanagridi, anche più dei Coliidi rispetto ai Fringillidi.

Viene quindi. la completa enumerazione degli splendienti Callocoraci: Cotingidi, Pipridi e Eurilaimidi, cui crediam me- 
glio spiccar dai Muscivori anche perchè baccivori; e li uniamo cioè ai Pittidi, Coraciilli e Prionitidi. Seguitano i Gressorii a piedi sindattili, in cui si hanno quasi trenta Meropidi, ed oltre a cento Alcedinidi. Saltiamo le due modificate stirpi degl'Insettivori e Larvivori, o per meglió dire diamo solo l'ultima famiglia, quella dei Dendrocolaptidi, con le sue ottanta specie tutte delle più calde parti di America abitatrici. Chiudiamo la Memoria con la poco numerosa stirpe dei Tenuirostri, tuttavia forse artifiziale malgrado l'abbia io tanto ristretta : e con questa si arriva ai Trochili già pubblicati.

\section{VOLUCRU M Cohors 2. A N ISODACTYLI.}

\section{AVIUM PASSERUM Stirps 11. FRUGIVORI.}

Familia 52. Bucerotide.

Subfamil. 135. Bucerotince.

a. Bucorveæ.

1. Bucorvus, Less. - 1. abyssinicus, $G m$. (caruuculatus, $W a g l$.) -2 ? leadbeateri, Vig. (cafer, Verr.)

\section{b. Buceroteæ.}

2. Ceratogyuna, $B p$. - 3. elata, Temm.

3. Tuetoceros, Caban. - 4. atratus, Temm. (ruficristalus, Temm. fæm.)5. cristatus, Rupp. - 6. cylindricus, Temm. - 7. buccinator, Temm. - 8. fistulator, Cassin. - 9. cultratus, Sundev.

4. Berenicornis, Bp. - 10. comatus, Raffles. (lugubris, Begbie.) 11. macrourus, Temm. (albicristatus, Cassin.)

5. Bucerotorus, Bp. (Cranocerus, Reich.) - 12. galeatus, Gm.

6. Buceros, L. - 13. rhinoceros, L. (sumatranus.) - 14. rhinoceroides, Temm. (bornensis.) - 15. sublunatus, Temm. (indicus.) - 16. lunatus, Temm. (javanicus. - sylvestris, Less. an Vieill.?)

7. Homraius, Bp. - 17. bicornis, L. (cristatus, Vieill.)

8. Hydrocorax, Briss. - 18. planicornis, Merr. (platyrhynchus, Pearson.)

9. Hydrocissa, Bp. - 19. monoceros, Shaw. - 20. pica, Scopoli. (malabaricus, $\mathrm{Gm}$.)

a. Anthracoceros, Reich. - 21 ? bicolor, Eyton. - 22. galeritus, Temm.

b. Anorrhinus, Reich. - 23. violaceus, Shaw. - 24. malayanus, Raffles. (anthraciuss, Temm.) - 25. exaratus, Reinw. 


\section{$-3-$}

10. RHYTICEROS, Reich.

a. Aceros, Hodgs. - 26. nepalensis, Hodgs.

b. Cassidix, $B p .-27$. cassidix, Ternm. (typicus, Bp.) - 28. corrugatus, Tenim. (gracilis, Temm. - rugosus, Begbie.)

c. Thyticeros, Bp. - 29. plicatus, Lath. - 30. ruficollis, Vieill: (pausuaran, Raffles.)

c. Tockeæ.

11. CalaO, Bp. - 31. sulcatus, Reinw.

12. Penelopides, Reich. - 32. sulcirostris, Wagl. - 33. panayensis, Scopoli.

13. Meniceros, Gloger. - 34. ginginianus, Lath.

14. Rhinoplax, Gloger.- 35. griseus, Lath - - 36. gingalensis, Shaw.

15. Grammicus, Reich. - 37. fasciatus, Shaw. - 38. nasutus, L. 39 ? hastatus, Cuv. (Levaill., t. 237.) - 40. limbatus, Rupp.

16. Rнулсносевоs, Gloger.- 41. melanoleucus, Licht. (coronatus, Shaw.)

17. Tockus, Less. - 42. flavirostris, Rupp. - 43. erythrorhynchus, Temm. (pæcilorhynchus, Lafr.)

Subfam. 136. Eurycerotince.

18. Edryceros, Less. - 44. prevosti, Less.

Familia 53. MusOPHAGIDE.

Subfam. 137. Musophagince.

19. Musophaga, 1 sert. - 45. violacea, 1 sert.

20. Gallirex, Less. - 46. gigantea, Vieill. - 47. rossæ, Gould. 48. porphyreolopha, $V i g$.

21. Turacus, Cuv. - 49. persa, L. (macrorhynchus, Fraser.) - 50. albicristatus, Strickl. - 51. buffoni, Vieill.

22. Opethus, Vieill. - 52. erythrolophos, Vieill. (paulina, Temm.)

23. Corythaix, Ill. - 53. leucotis, Ill.

24. Coliphimus, Smith. -54 . concolor, Smith. (feliciæ, Less.)

25. Schizorrhis, Wagl. - 55. leucogastra, Rupp. - 56. personata, Rupp. - 57. zonura, Rupp. - 58. africana, Lath. (variegata, Vieill.)

Familia 54. CoLIIDE.

Subfam. 138. Coliince.

26. Urocolius, $B p$. -59 . macrourus, $L$. (senegalensis, $G m$.) -60 . indicus, Lath. (quiriva, Less.)

27. Colius, $B r$. -61 . capensis, Gm. (L. colius, L.)

28. RhaBdocolius, $B p$. - 62 . striatus, $G m$. (panayensis, $G m$.) - 63. nigricollis? Vieill. - 64. leucotis, Rupp. 
Familia 55. OPISTHOconı...

Subfam. 139. Opisthocomince.

29. Opisthoconus, Vieill. -65 . cristatus, Gm.

Familia 56. Phytotonid.E.

Subfam. 140. Phytotomince.

30. Phyтотомa, Molina. - 66. rara, Molina. (silens, Kiltl. - bloxami, Childr. - molinæ, Less.) - 67. angustirostris, Orb. - 68. rutila, Vieill.

Stirps 12. CALLOCORACES.

Familia 57. Cotingid.E.

Subfam. 141. Lipaugince.

31. Lipadgus, Boie. -69. plumbeus, Licht.-70. fusco-cinereus, Lafr

32. Lathrid, Sw. - 71. simplex, Licht.

33. Aulea, Schiff. - 72. hypopyrrha, Vieill. (sibilatrix, Wied. - sanguinaria, Less.) - 73 ? lanioides, Less.

34. SChIFForis, Bp.-74. major, Bp.-75. turdina, Wied.-76. minor, Schiff.

35. Heteropelia. Schiff. - 77. viridis, Less. (rufo-olivacea, Lafr.) 78. unicolor, Ménétr. (vireșcens, Lafr.)

Subfam. 142. Querulince.

36. Pyroderus, Gr. - 79. scutatus, Shau. - 80. granadensis, Lafr. 81. orenocensis, Lafr.

37. Qeerdia, Vieill. - 82. rubricollis, Gm.

33. He.matoderus, Bp. - 83. militaris, Gm.

Subfam. 143. Gymnoderince.

39. Gimocerhatus, Geoffr. -84 . calvus, $G m$.

40. CFiphaloptrites, Geoffr. -85 . ornatus, Geoffr. -86 . glabricollis, Gould.

41. Gyuxodercs, Geoffr. -87 . foetidus, $L$.

42. Chasmorhyschus, Temm. -88 . variegatus, $L$.

43. Arapuiga, Less. - 89. alba, Thunberg. - 90. carunculata, Gm. -91. tricarunculata, Verr. 


\section{Sub'am. 14\}. Cotingina. \\ a. Cotingeæ.}

44. Py ronhyсches, Lafr. - 92. viridis, Orb. - 93. riefferi, Boissonn. -.94. arcuatus, Lafr.

45. Euchlonns, De Filippi. - 95. aureipectus, Lafr. (elegans. Tsch.) - 96. formosa, Hartl. - 97 sclateri, Cornelia. - 98? cincta, T'schudi. (tschudii, Gray.) - 94. melanolæma, Sclater.

46. Ptilochloris, Sw. - 100. arcuatus, Geoffr. - 101. remigialis, Lafr.

47. Tijucia, Less. - 102. nigra, Less.

48. Aypelion, Caban. - 103. melanocephala, Vieill. - 104. cucullata, $S w$.

49. Heliochera, De Filippi. - 10j. rubrocristata, Lafresnaye. 106 ? rufaxilla, Tschudi.

50. Phibalura, Vieill. - 107. flavirostris, Vieill.

51. Xipholesa, Gloger. - 108. pompadora, L. - 109. atropurpurea Wied. - 110. lamellipennis, Lafr.

52. Cotinga, Br. - 111. cærulea, Vieill. (cælestis, Bp. Consp.) 112. nattereri, Boissonn. - 113. cincta, Gr. (cærulea, Bp. nec Vieill.) 114. cayana, L. - 115. maynana, $L$.

53. Porphyrol eis , Bp. - 116. phygas, $B p$. (Cotinga porphyrolæma, Sclater.)

b. Iodopleureæ.

5ł. IOdOPleurA, Lesson. - 117. fusca, Vieillot. (Japlacii, Eydoux.) 118. isabellæ, Parzudaki (guttatı, Less.) - 119. pipra, Less.

Familia 58. PIPRIDE.

Subfam. 145. Rupicolince.

5j. Rupicola, Br. - 120. crocea, L. - 121. peruviana, Lath.

56. Ph.enicocercus, $S w$. - 122. carnifex, $L$. - 123. nigricollis, $S w$.

Subfam. 146. Piprince.

57. Axtilophia, Reich. - 124. galeata, Licht.

58. Masius, Bp. - 125. chrysopterus, Lafr.

59. Manacus, $B r$. - 126. gutturosus, Desmar. - 127. cdwardsi, $B p$. - 128. flaveolus, Cassin. (hogotensis? Sclater.) - 129. candei, Parzudaki. - 130. vitellinus, Gould, - 131. flavitinctus, Sclater.

60. Xenopipo, Caban. - 132. atronitens, Caban.

61. Chiroprion, Schiff. - 133. pareola, $L$.

62. Chiroxıphia, Caban. - 134. ignicapilla, Wagl. - 135. caudata, Shav. - 136. longicauda, Vieill. - 137. melanocephala, Vieill. 
63. Gercophana, Schiff. - 138. linearis, Bp.

64. Hicura, Reich. - 139. militaris, Shaw.

65. Cirripipra, Bp. (Teleonema, Reich.) - 140. filicauda, Spix.

66. Pipra, Linn. - 141. aureola, L. - 142. aurantia, Wagler. 143. fasciata, Orb. - 144. flavicollis, Sclater.

67. Dixiphia, Reich. - 145. erythrocephala, L. - 146. chloromeros, Tschudi. - 147. rubricapilla, Brisson. - 148. leucocapilla, Linn. 149. cæruleocapilla, Tschudi.

68. LePidothrix, Schiff.-150. cyaneocapilla, Wagl. (herbacea, Spix, frem.) - 151. isidorii, Sclater.

69. Ceratopipra, Bp. - 152. cornuta, Spix.

70. Corapipo, Schiff. - 153. gutturalis, L. (perspicillata, Wagl. ææm.)

71. Dasyncetopa, Schiff. - 154. serena, $L$.

72. Macheropterus, Schiff. - 155. strigilatus, Pr. Max. Wied. 156. striolatus, $B p .-157$. pyrocephalus, Sclat.

73. Hemipipo, Caban. - 158. chloris, Natter. - 159. chlorion, Caban.

74. Piprites, Caban. - 160. pileata, Natter. - 161. flavicapilla, Sclater.

75, Calypturus, Sw. - 162. cristatus, Vieill.

Familia 59. EURYLEMIDE.

Subfam. 147. Calyptomanince.

76. Galyptomena, Raffles. - 163. viridis, Raffles.

Subfam. 148. Eurylæemince.

77. Peltops, Wagl. - 164. blainvillii, Garnot.

78. Parisonus, $S w .-165$. dalhousiæ, Jameson.

79. Serilophus, $S w .-166$. lunatus, Gould. -167 . rubropygius, Hodgs.

80. Gymbir hynchus, Vig. - 168. macrorhynchus, Gm. - 169. affinis, Blyth.

81. Eurylemus, Horsf. - 170. javanus, Horsf. - 171. ochromalus, Raffles.

82. Corydon, Less. - 172. sumatranus, Raftes.

Subfam. 149. Smithornithince.

83. Suithornis, $B p .-173$. capensis, Smith.

Familia 60: PITTIDe.

Subfam. 150. Pittince.

84. Brachyorus, Thunb. (Pitta, Reich.) 
a. Gigantipitta, Bp. - 174. cærulea, Raffles. (gigas, Temm.) 175. cyanea, Blyth. (olim gigas.) - 176. nepalensis, Hodgs. (nuchalis, Blyth.) - 177. maxima, F'orst.

b. Brachyurus, Bp. - 178. strepitans, Temm. (versicolor, $S_{w .}$.) 179. irena. Temm. - 180. cyanoptera, Temm. - 181. malaccen. sis, Scopoli. - 182. bengalensis, Gm. (Corvus brachyurus, L.) 183. nympha, Schlegel. - 184. maculata, Temm. - 185. vigorsi, Gould. - 186. angolensis, Vieill. (pulih 3 Fraser.)

c. Erythropitta, Bp. - 187. mackloti, Temm. - 188. celebensis, Forst. - 189. erythrogastra, Cuv. - 190. venusta, Mull. 191. granatina, T'emm. (coccinea, Eyt.)

d. Iridipitta, Bp. - 192. baudi, Mull. - 193. iris, Gould.

e. Melanopitta, Bp. - 194. cucullata, Hartl. - 195. forsteni, Bp.196. mulleri, Bp. - 197. novæ-guineæ, Schlegel. - 198. atricapilla, Cuv.

85. Pitta, Vieill. (Eucycla, Reichenbach.) - 199. cyanura, Gmelin. 200. schwaneri, Temm. - 201. elegans, Temm. (nec irena, Temm. boschi, Mull. et Schleg.)

\section{Familia 61. CoraciIde.}

Subfam. 151. Coraciince.

86. Coraciora, Bp. - 202. cyanogastra, Cuv. - 203. caudata, L. (angolensis, Br. - natalensis, Licht.) - 204. abyssinica, Gm. (senegalensis, Gm.)

87. Coracias, $L$. - 205. garrula, $L$. -206 . indica, $L$. (viridis ? Cuv.) 207. bengalensis, L. (alfinis, Mac Clell.) - *208. crinita, Shaw. (pilosa, Lath. ex Afr. m.) - 209. nuchalis, Sw. (Ievaillantii, Rupp. - pilosa, Hartl.) - 210. pileata, Reinw. (temmincki, Vieill. - d'urvillii, Quoy et Gaim.)

88. Colaris, Cuv. - 211. orientalis, L. - 212. cyanicollis, Vieill. 213. pacificus, Lath.

89. Eurystonus, Vieill. - 214. madagascariensis, Gm. (violaceus, Vieill.) -215. gularis, Vieill. - 216. purpurascens, Vieill. - 217. afer, Lath. (viridis, Wagl. jun.)

Subfarn, 152. Atelornithince.

90. Atelornis, Pucheran. - 218. squamigera, Lafresn.

91. Brachypteracias, Lafr. (Chloropygia, $S_{w .}$ ) - 219. leptosoma, Less.

92. Corapitta, Verr. - 220. piltoides, Lafr.

Familia 62. Prionitide.

Subfam. 153. Prionitince.

93. CR YPticus, Sw. - 221. superciliaris, Sandback. (yucatanensis, Cabot.) 222. martii, Spix. (plathyrhynchus, Leadb.) - 223. carinatus, Dubus.

94. Prionites, Ill.

a. Momotus, $B p$. ex $B r$. -224 . mexicanus, $S w$. 
b) Raryphonus, Bp. ex Vieill.-225. semirufus, Sclater. - 226. ruficapillus, Ill. et Vieill. (I.vaillantii, I.ess. - dombeyanus, Ranz.)

C. Prionites, $\boldsymbol{B}_{\text {p }}$. ex $\mathbf{I l l}$. - 227. subrufescens, Sclater. - 228. bahauneusis, Su. - 299. psalurus, Pucheran. (subhutu ? Less.) - 230. cæruleiceps, Gould. -..- 231 ? parvirostris, Bonctp. ex Carthagena. 232. momota, L. (brasilicusis, Lath. - cyanocephaluc, Vieill. ex Cayenna.) 233. gularis, Lafr.

9j. II lomaxes, licht. - 234. momotula, Licht.

\section{Stirps 13. GRESSORI (Syndactyli).}

Familia 63. MEroIID£.

Subfam. 154. Meropince.

96. Merons, $L$.

a. Мегореж.

a. Merops, Reich. - 235. apiaster, L.

J. Blepharomerops, Reich. - 236. ægyptius, Forsh. (persicus, Pall.)237. savignyi, S $w$. - 238. vaillantii, $B$ p. - 239. superciliosus, $L$. - 240. javanicus, Horsf. - 2\$1. typicus, Hodgs. (philippinus, Auct. nec $L$. - sarignyoides, Mus. Mass.)

c. Melittophas, Reich. - 242. philippinus, L. (bodins, Gm. ad. - adansoni, Lev. et senegulensis, Shaw. fictitia avis! - hypoglaurus, Reich. jun.)

d. Aerops, Reich. - 243. albicollis, Vieill. (cuvieri, Licht.)

e. Urica, Bp. - 244. ornatus, Lath. - 245. quinticolor, Vieill. 246. leschenaulti, Vieill. (urica, Horsf.-sumatranus, Faffles.)

97. Phloturus, Reich. - 247. viridis, L. (1amarchi, Cuv.) - 248. viridissimus, $S u$.

93. Inlttotheres, Reich. - 249. nubicus, Gm. - 250. nubicoides, O. des Wurs et Pucher. (nataleusis, Reich.)

99. Tephraerops, Reich. - 251. bicolor, Duud.

100. Melittophagls, Boie. - 252. hirundinaceus, Vieill.

101. SPHEсорновтs, Reichenbach. - 253. erythropterus, Limn. 25\%. variegatus, Fieill. (souniui, $V_{\text {. }}-$ cullanis, $V_{\text {. }}$ - cyanipectus, Verr.) 255. lafresnayii, Guér. (lefebrre. O. des Murs.)

\section{b. Nyctiornithinæ.}

102. Coccolarixx, Reich. - 250. bullocki, Vieill. - 257. bullockoides, Smith.

103. Neropiscus, Sundev. - 258. gularis, Lath.

i04. Neropogox, Bp. - 259. forsteni, Temm.

10j. Nictiornis, $S w$. (Alcemerops, Is. Geoffr.)

a. Nyctiornis, $S w$. -260 . amictus, Temm.

b. Bucia, Hodlgs. - 261. athertoni, Jardine. 
Familia 64. .JLCEDinde.

Subfanı. 15j. Dacelinue.

106. Dacelo, Leach. - 262. gigantea, Lath. - 263. leachi, Lath. (cervina, Gould.)

107. Choccalcros, Bp. ex Less. (Monachalcyou, Reich.) - 264. gaudichaudi, Quoy et Gaim.

108. Melidora, Less. - 265. cuphrasiæ, Less.

109. Syya, Less. - 266. torotoro, Less. - 267. tlavirostris, Gould.

110. Paralcyox, Bp. ex Gloger. - 268. concretus, Temm. - 269. monachus, Forsten. (princeps, Forst.) - 270. lindsayi, Vig.

111. Lacedo, Reich. - 271. pulchella, Horsf. (buccoides, T'emm.) 272. melanops, Temm.

112. Acteroides, Hombr. - 273. hombroni, Bp. (variegalus, Pucheran.)

113. Cittrira, Kaup. - 274. cyanotis, Temm.

114. Chelicutra, Reich. - 275. pygmæa, Cret:schm. - 276. striolata, Licht. - 277. fuscicapilla, Lafr. (vaillanti, Temm.)

Subfam. 156. Halcyonince.

115. Callálctos, Bp. - 278. coromanda, Lath. - 279? lilacina, Bp. - 280. schlegeli, $B p$.

116. Cancrophaga, Bp. - 281. badia, Ferr. - 282. lathami, $B p$. (cancrophaga, Lath.) - 283. semicærulea, Gm. - 28٪. malimbica, Shaw. - :85. rufirentris, Sw. - 286. senegalensis, L. - 287. senegaloides, Smith. - 288, cinereifrons, Vicill. - 289. dryas, Hartl. 290. cyanoleuca, Vieill.

117. Halcyox, Sw. - 291. cyaniventris, Vieill. (omuicolor, Reinw.) 292. gularis, Kuhl. (fusca, Gray et Bodd.) - 293. smyrnensis, L. 294. atricapilla, Gm.

118. Todiramphus, Less, - 29j. albicilla, Cuv. - 296. saurophaga, Gould. - 297. divinus, Less. - 298. sacer, Gm. - 399. platyrostris, Gould. - 300. sancta, Vig. et Horsf. - 301. venerata, Gm. 302. varia, Eyton. - 303. ruficeps, Cuv. (cinnamomina, $S_{w}$.) -304 . vagans, Less. - 305. sordida, Gould. - 306. coronala, Mull. (australasia ! Vieill.) - 307. collaris, Sw. - 308. chlorocephala, Gm.

119. Gyanalcyox, Verr. - 309. pyrrhopygia, Gould. - 310. macleayi, Jard. - 311. lazuli, Temm. (diops, Temm.) - *312. lazulin us, Schiff. - 313. forsteni, Temm. - 314. funebris, Temm.

120. Taymsiptera, Vig. - 315. dea, L. - 316. sylvia, Gould.

121. Ispidint, Kaup. - 317. cárulea, Gm. (cyanotis, Sw.) - 318. madagascariensis, $\mathrm{Br}$.

122. Ceyx, Lacép. - 319. tridactyla, L. - 320. purpurea, Gm, 321. rufidorsa, Strickl. - 322. melanura, Kaup. - 323. lepida, Temm. 
123. Ramphalcyon, Reich.

a. Hylcaon, Reich. - 324. melanorhynchus, Temm.

b. Ramphalcyon, Reich. - 325. leucocephala, Gm. - 326. javanica, Shaw. -327. gurial, Pears. (capensis ! L.) - 328. amauroptera, Pears.

Subfam. 157. Alcedinince.

a. Ceryleæ.

12^. MegaCeryle, Reich. - 329. maxima, Pall. (guttata, Bodd. - gigantea, $\left.S_{w_{0}}\right)$ - 330. lugubris, Temm. - 331. guttata, Vig.

125. Streptoceryle, Bp. - 332. torquata, L. (stellata, Reich. - domin gensis, Reich.) -333 . alcyon, $L$.

126. Ceryle, Boie. - 334 rudis, $L$. (bicincla, $S w$. mas.) -335 ? varia, Strickl. (leucomelanura? Reich.)

127. Chloroceryle, Kaup. - 336. amazona, Gm. vestita? Cuv. leucosticta, Reich.) - 337. americana, Gm. (cabanisi, Tschudi.)

128. Amazoxis, Reich. - 338. bicolor, Gm. - 339. superciliosa, $L$.

b. Alcedineæ.

129. Alcedo, $L .-340$. ispida, $L .-341$. ispidoides, Less. -342 . ja-

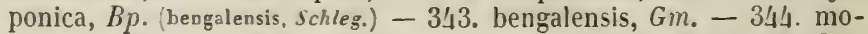
luccensis, Blyth. -345 . euryzona, Temm. -346 . semitorquata, Sw. (azurea ? Less) -3 '7. cærulescens, Vieill. (birn, Horsf.) - 348. meninting. Horsf. -349 . verreauxi, Temm -350 . quadribrachys, Temm. - 351. megarhỵnchus, Schiff.

130. Corythorsis, Kaup. - 352. leucogastra, Fraser. - 353. nitida, Kaup - 35 ? cæruleocephala, Gm. - 355. cristata, L. - 356. nais, Kaup. - 3j7. vintsioides, Eyd. et Gerv.

131. Alcroxe, Swainson. (Therosa, Mull.) - 358. solitaria, Temm. 359. cyanipectus, Lafr. (cincta ? Jurdine.) - 360. pusilla, Temm. 361. azurea, Latham. - 362. tribrachys, Temminck. -363 ? lessoni, Cassin, Pr. Ac. Phil., v. p. 69.

Huc Stirps 14. INSECTIVORI. - Fam. 65. TODIDE.

Fam. 66. Mexuride. - Fam. 67. Myiotheride. et

Stirps 15. LARVIVORI. - Fam. 68 ANaBatid.E.

Fam. 69. Dexdrocolaptide.

Subfam. 170. Dendrocolaptince.

a. Dendrocolapteæ.

1. Dendrocolaptes, Hermann.

a. Dendrocolaptes, Auct. - 1. major, Vieill. (rubiginosus, Lafr. - falcirostris ? Spix.) - 2. albicollis, Vieill. - 3. simpliciceps, Puch.

b. Xiphocolaptes, Less. - 4. promeropirhynchus, Less. 
2. Cladoscopos, Reich. - 5. perroti, Lafr. - 6. temmincki, Lafr. 7. devillii, 0 . des Murs.

3. LEPIDOCOLAPTES, Reich. - 8. squamatus, Licht. (levaillantii, Reich.) 9. wagleri, Spix. - 10. leucogaster, Sw. - 11. atripes, Eyton. *12. delatrii, Bp. - 13. souleyeti, Lafr. - 11. lineaticeps, Lafr. 15. albilineatus, Lafr. - 16. validirostris, Eyton. - 17. fuscus? vel notatus? Eyton.

4. Picolaptes, Less. - 18. tenuirostris, Licht. (spixi? Less.) - *19. chionolæma, Bp. - 20. bivittatus, Lafr. - 21. angustirostris, Vieill.

5. DaCryophorus, Bp. - 22. lacrymiger, Lafr. - 23. affinis, Lafr.

6. Хірновнуnchus, Sw. -24 . trochilirostris, Licht. (falcularius? Vieill.) - 25. procurvus, Temm. -26 . procurvoides, Lafr. - 27. lafresnayanus, Orb. - *28. granadensis, Lafr. - *29. venezuelensis, Lafr. —*30. isabella, Verr. — *31 ? bre virostris, Lafr.

7. Drymornis, Eyton. - 32. pucherani, Lafr. - 33. bridgesi, Eyton.

8. NASICA, Less. - 34. longirostris, Licht. (rufigula, Less. - albicollis? Less.)

9. Dendrornis, Eyton. (Premnocopus, Caban.) - 35. guttatus, Licht. 36. pardalotus, Vieill. (flammeus, Licht.) -37 . guttatoides, Lafr. 38. flavigaster, $S w$. (eburneirostris, Less.) - 39. multiguttatus, Deville. - *40. rostripallens, Lafr. — *41. kieneri. Lafr. — *42. weddelli, Lafr. - 43, triangularis, Lafr. - 44. beauperthuyi, Pucheran. 45. dorbignyanus, Pucheran. - 46. chunchotumbo, Tschudi. 47. ocellatus, Tschudi. - 48. obsoletus, Illig. - 49. susurrans, Wagl. (albisquama, Lafr.)

10. Dendroplex, Sw. - 50. picus, Gm. - 51. picirostris, Lafr. *52? intermedius, $B p$.

11. NEOPS, Vieill. (Glyphorhynchus, Wied.) -- 53. cuneatus, Licht. 54. venezuelensis, Lafr. - 55. spirurus, Vieill. (Levaill., Promer. t. 31.) - *56. castelnaui, Lafr.

12. Sitrasomus, $S w .-57$. erythrurus, Licht. -58 . olivaceus, Wied. - 59. sylvioides, Lafr. - 60. amazonus, Deville. - 61. griseus, Jard. - 62. pallescens, Lafr. - *63 ? orbignyanus, Lafr.

\section{$b$. Dendrocopeæ.}

13. Dendrocops, $S w$. - 64. cayanensis, $G m$. - 65. platyrostris, Spix. -66 ? validus, Tschudi. -67 . crassirostris, Such. -68 ? fortirostris, Such. - 69. multistriatus, Eyton. - 70. sancti-thomæ, Lafr. - 71. olivaceus, Eyton.

14. Dendrocincla, Gr. - 72? tyrannina, Lafr. -73 . turdina, Licht. -74. fumigata, Licht. - 75 ? fuliginosa ? Less. - 76. atrirostris, Lafr. - 77. merula, Lafr. - 78. meruloides, Lafr.

15. Dendroxetastes, Eyton. - 79. capitoides, Eyton. 
Slimps 16. TEVUIROSTRES.

Familia 70. LP'P1D.E.

Subfam. 171. Upupince.

1. Upeps, L. - 1. epops, L. - 2 ? senegalensis, Sw. 3. minor, Shaw. (capensıs, siv) - *4. indica, Layard. - *5. madagascariensis, Bp. - *6. marginata, Peters.

Familia 71. Proueropid..

Subfam. 172. Falculiince.

2. Filculia, Is. Geoffr. - 7. palliata, Is. Geoffr.

3. Fregilupus, Less. - 8. capensis, Gm. (cristata, Vieill.)

Subfam. 173. Promeropince.

4. Proverops, Br. - 9. erythrorhynchus, Lath. (capensis, Less.) *10. stricklandi, Bp. ex Damara. - 11. melanorhynchus, Licht. (senegalensi-, Vieill.) - 12. caudacutus, Vieill. (abyssicicus, Verr:)

5. Irrisor, Less. - 13. pusillus, Sw. (aterrimus, steph.)

6. Rhryopomastes, Smith. - 1'. cyanomelas, Vieill. (smithi, Jardin.purpurea, Burch.) - 15. minor, Rupp.

(Huc Stirps 17. SLSPENSI sive TROCHILI.)

Cogliamo questa occasione per correggere alcuni errori corsi nel Quadro dei Volucri zigodattili. Per inavertenza inesplicabile il compositore ha aggiunto sotto i numeri 52 e 53 alla famiglia americana dei Raxfastid due specie crawfordi e javanicus trasportate dal genere lielias della famiglia dei Cuculidi, una delle quali si trova anche registrata al suo posto duplicatamente.

I Ranfastidi dunque terminano col numero 51, il quale è precisamente lo stesso di quello che dà Gould nella sua recentissima seconda edizione della Monografia di quegli uccelli. Questa coincidenza nasce dal non ammetter egli Pteroglossus ambiguus, Less., da me come specie dubbia registrato sotto il $n^{\circ} 20$, e ch'egli riunisce al regalis, Licht., sotto il nome di torquatus: mentre ne figura una nuovissima e bellissima sotto il nome di Aulacoramphus cceruleogularis, Gould. Si aggiunga dunque questa specie al mio Catalogo, avvertendo però che 
deve far parte del genere Ramphoxanthus cui appartengono altresi le specie 46. wagleri e 49. atrigular is dello Sturm., collocate a torto sotto Aulacoramphus.

Venendo ai Cuculid oltre la restituzione già indicata da farsi al genere Melias, si noti che la specie 198 dev'essere Chrysococcyx klaas (non klausii) dal nome del fedele caffro del Levaillant.

Non saprei abbastanza inculcare scrupolosi studj circa le varie specie di Nicoclarius e di Cacomantis, e sopratutto sui pretesi Cuculus sonnerati di Latham, varius di Vahl, micropterus di Gould e il recentissimo bartlettii di Lavard, senz'altro il giovane di altra specie registrata.

Osserviamo nei veri Scassori, ossia Picchj, cle il mio Picus kamtschatschensis è troppo sinile al minor, L., per non far parte dello stesso sotto-genere Pipripicus. E già si sa clie questa ultima specie, come si può vedere nell'ammirabile serie messa insieme dal signor Malherbe, varia nelle diverse località. Più piccola e più nera inferiormente in Algeria, mostrasi in rece in Norvegia più grande e con più bianco, non mai però tanto quanto nel kamtschatschensis.

Arendo elevato a grado di specie alcune razze di Micropterni sarà forse bene di distinguere anche quella dell'isola di Ceilan sotto il nome di Micropternus layardi, Bp.

Il genere 19. Chrysopicus, Malherbe, è meglio riguardarlo come sotto-genere di Chloronerpes, Swains., il 22. Capnopicus sarà forse meglio considerarlo come terzo sotto-genere di Veniliornis. Aggiungi alle specie del secondo, Eleopicus, un nuovo picchio che il Nalherbe va a descrivere sotto il nome di Mesopicus primolia dedicandolo gentilmente, dal maritale cognome, a questa amatissima mia seconda figlia Carlotta.

Questo Picologo dottrinatissimo la fatto molti e giusti rilieri circa i miei Chrisoptilei africani : la specie 127. obsoletus, Wagler, è un Dendropicus non un Dendromus. La specie 117. cardinalis, Gmelin, presa dalla figura 35 del viaggio alla nuova Guinea del Sonnerat non differisce dalla 115. fuscescens ossia 
fulviscapus, detta dell'isola di Luçon per un errore che frequente incontrasi nell'assegnare la patria. Lo stesso accadde al Somnerat per il suo Picchio verde della predetta isola, tavola 36 , che altro non è se non la femmina del Mesopicus capensis. E quì si noti che il poliocephalus di Cuvier, anch'esso del Senegal, si riferisce al pari di quello di Swainson al goertan di Gmelin. La specie 126. rufo-viridis, Malherbe, si abolisca, perche è la femmina del 130. brachyrhynchus, Swains., e questa fusione si trasferisca al genere Pardipicus. Si cancelli pure dal numero degli esseri la specie 131. guttatus ossia variolosus di Lichtenstein, che altro non è che un sinonimo della 123. bennetti, Smith. L'œthiopicus di Hemprich ed Ehrenberg, figurata dal Rüppel, è indubitatamente il vero nubicus di Gmelin, specie che varia molto, e di cui la Planche enluminée 667 di Buffon rappresenta la femmina.

Così i miei tredici Dendromi si riducano ad otto; tre essendo specie nominali, uno Dendropicus e l'altro Pardipicus. È quası soverchio il dire clie il nome di nibicus va esclusivamente conservato a quello di Gmelin e di Buffon, al quale lo restituì lo stesso Lichtenstein nel 1840; che per conseguenza il notatus, Lichtenstein, del capo di Buona-Speranza figurato da Levaillant alla tavola 250, non è il nubicus di Gmelin, quantunque sia quello di Vieillot, di Valencienres, di Wagler, e del mio Conspectus; e che il nubicus, Licht. del 1823 deve chiamarsi esclusivamente punctuligerus, Wagler. Lo smithi, Malherbe, è sinomino del chrysurus, Swains., non dell'abingoni, Smith.

Al genere 24. Brachypternus aggiungi una nuova bellissima specie Brachypternus stricklandi, Layard (rubescens? Kelaart.) dell'isola di Ceilan : Castaneo-purpureus plumis purpureo-marginatis: uropygio coccineo: subtus cervinus, juguli pectorisque plumis medio albis, margine nigro tamquam squamatus : gula albalineis quinque fuscis: cauda, cum remigibus albo-maculatis, brunnea.

Mas. Pileo rubro.

Fæm. Pileo fusco albo-margaritato.

I generi 29 e 30 differiscono poco l'uno dall'altro. 
Passando alla stirpe dei Barbatı poco abbiam che dire circa i BucConidi se non che l'aurifrons di Vigors, e il glaucogularis di Tschudi sono veri Eubucco non Micropogon. Moltissimo poi avrem da rilevare circa i CAPITONIDI, atteso che mentre noi stampavamo anche il signor Sclater pubblicava una eccellente Memoria sui medesimi, nella quale non dà solamente i nomi ma anche i caratteri delle sue numerose specie. Il signor Sclater li chiama Bucconıd, e quel che è più ne rompe le affinità coi miei Bucconidi, ch'egli chiama Capitonidi, collocandoli fra i FissiRostri. Del resto i suoi generi corrispondono presso a poco ai miei; soltanto T'amatia, Chaunornis e Cyphos sono sottogeneri di Bucco (ch'è il mio Capito). Egli chiama Nonnula il gruppo cui ho applicato il nome Scotocharis, Gloger, e lo fa sottogenere di Malacoptila. Non adotta affatto il mio Nyctastes che riunisce a Chaunornis.

Veniamo alla rivista delle specie. Costituisce il suo Bucco (Capito) del solo capensis di Linneo. Non conosce il mio Tamatia hyperrhynchus nè il mio gigas : ma agli altri miei quattro ągiunge il melanoleucus ossia tectus che è per me un Capito. Primo dei suoi Chaunornis è il tamatia, Gmelin, ch'io pongo pure fra i Capito. Vi pone lu Sclater altresì il lanceolatus, De ville, e il chacuru, Vieillot, che è per me un Nyctastes, come pure il maculatus, Gmelin, e lo striatipectus, che non differisce dal flammulatus, Verreaux, al quale aggiunge come specie nuova il suo radiatus chè forse il mio ruficervix. Dubita essere buona specie la Malacoptila inornata, Dubus; registra in questo genere la panamensis, Lafresnaye; la rufa, Spix, e la mystacalis, Lafresnaye, delle quali io faceva Scotocharis : chiama fulvogularis la mia pyrrholama, e due nuove del tutto ne aggiunge: Malacoptila substriata, ed aspersa, Sclater, proveniente la prima dalla nuova Granata, da Caraccas in Venezuela la seconda. Egli ha così nove Malacoptile in vece delle mie tre; non rimanendo in Scotocharis (Nonnula) che la rubecula e la ruficapilla, cui aggiunge come specie intermedia, la sua frontalis di Santa-Fé di Bogota. Quattro sono come nel mio Quadro le vere Monase. 
Le stessissime mie, per le quali pero adotta diversi nomi chiamando atra la mia tranquilla; flavirostris, l'axillaris; e forse non senza ragione, personata, Vieillot, la leucops di Lichtenstein.

Lo stesso signor Sclater mi ha farorito alcuni rilievi ed un perfettissimo indice manoscritto della famiglia dei GaLBULId, in cui dubita, ma credo a torto, della validità della specie Galbula quadricolor di Verreaux, ed in cui mi rimprovera di non aver badato ad un articolo del Cabanis, ch'io non conosco. Vuole con ragione più rarricinate le quattro specie viridis, melanogeniu, maculicauda e ruficauda, che non differiscono fra loro se non per la colorazione della coda. Mi arverte che Galbula chalcocephala, Deville, contrariamente alla stessa sua opinione anteriore, non è la medesima di leucogastra, Vieillot, ma bensì una specie molto più prossima all'albirostris. Asserisce, per averlo verificato in Nonaco sull'esemplare originale, che l'albigularis di Spix (cui egli prende a torto per il tipo del mio genere $\mathrm{Bra}$ chigalba) non è che il giovine dell'Urogalba paradisea. Osserra che la sua inornata, vero tipo del mio genere e che ammette essere la lugubris di Cabanis e la chalcoptera di Reichenbach, nulla ha che fare con l'albiventer, Curier. Fondata questa neI 1829, Regno animale, I, p. 442, sulla figura di Levaillant, mal citata da esso Curier; che avrebbe dovuto scrivere Levaill. Suppl. aux Jacamars dans le vol. des Guêpiers, fig. h. non differisce dalla leucogastra. Aggiunge finalemente una nuova specie affine proveniente dal Rio Napo che chiama Galbula chalcothorax.

Poco o nulla abbiam da aggiungere ai Trogosid : diciam soltanto che il nome di fasciatus, Gmelin, deve indubitatamente restituirsi all' Harpactes malabaricus, Swains. essendo più antico di esso e comunissimo nell'isola di Ceilan. Per la stessa ragione deve chiamarsi kasumba, Raffles, la specie affine di Sumatra e della penisola di Malacca.

(Estratto dall'Ateneo italiano, $\mathrm{n}^{\circ} 11$, agosto 1854.)

Parigi, - Dalla stamperia di I.. MARTiNeT, via Mignon, 2. 
1.

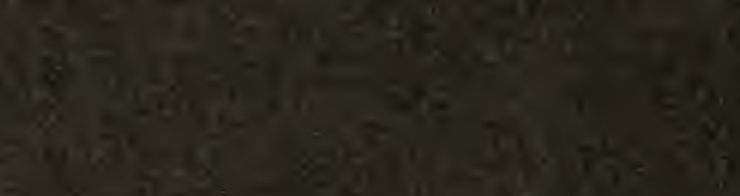

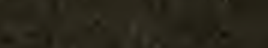

